\title{
Cervical neoplasia and human papilloma virus infection in prostitutes
}

\author{
G Gitsch, Ch. Kainz, A Reinthaller, W Kopp, G Tatra, G Breitenecker
}

\begin{abstract}
Objectives-To evaluate the prevalence and incidence of PAP smears indicating cervical dysplasia as well as human papillomavirus (HPV) infection in prostitutes.

Design-Prevalence and incidence study of cervical dysplasia and HPV infection in prostitutes. For detection and typing of HPV-DNA In Situ Hybridisation (ISH) was performed in tissue samples with CIN gained by colposcopically directed punch biopsies.

Setting-Second Department of Obstetrics and Gynecology, University of Vienna Medical School and STD Clinic of the Public Health Office, Vienna.

Subjects-Registered prostitutes attending the STD Clinic of the Public Health Office and a control group.

Results $\longrightarrow 978$ prostitutes and 5493 women with unknown cytological anamnesis were compared. Frequency of positive PAP smears was significantly higher in prostitutes $(6 \cdot 13 \%$ versus $1 \cdot 43 \%$ ). To determine the pick-up rate of cervical dysplasia during one year after negative cytology we compared 722 prostitutes and 3162 controls. Prostitutes showed a significant higher dysplasia pick-up rate $(3.05 \%$ to $1.07 \%$ ) compared with controls. HPV detection rate in prostitutes was similar to that in the control group. The distribution of HPV types revealed a higher frequency of "high risk" HPV 16/18 and 31/33 in prostitutes.

Conclusion-The results demonstrate a higher incidence and prevalence of cervical dysplasia in prostitutes and therefore suggest regular cervical PAP smear screening in registered prostitutes twice a year.
\end{abstract}

Second Department of Obstetrics and Gynecology, University of Vienna, Austria

G Gitsch, Ch. Kainz, A Reinthaller, W Kopp, G Tatra, G Breitenecker
Introduction

Many different factors are supposed to play a role in natural history of cervical intraepithelial neoplasia (CIN) and invasive cervical cancer. ${ }^{1-3}$ Epidemiological studies have shown that early sexual contact, high promiscuity and low social status are important causes for the development of cervical neoplasia. ${ }^{45}$ Results of recent studies concerning frequency of CIN in prostitutes compared with a normal population are different. Schuhmann described the same frequency of dysplasia in both groups, while others evaluated a range up to a tenfold higher frequency of CIN in prostitutes. ${ }^{6-8}$ In our study, we compared the prevalence of cervical dysplasia in prostitutes and in a control group, and we explored the incidence of PAP smears indicating cervical dysplasia one year after normal cytology in both groups.

During the last years the importance of human papillomavirus (HPV) infection for the generation of CIN has been recognised..$^{910}$ The observation that certain HPV types are associated with different grades of CIN and different progression rates of CIN prompted us to determine the HPV infection rate and distribution of HPV types 6/11, 16/18 and 31/33 in colposcopically directed punch biopsies from cervical lesions of prostitutes and controls. ${ }^{112}$

\section{Material and methods}

Patients: In Vienna, Austria, prostitutes by law must register and undergo weekly medical examinations in the STD Clinic of the Public Health Office. Registered prostitutes (978) were included in the study. Routine cervical cytology was taken in addition to STD screening for the first time in 1988. As a control group we evaluated PAP smears of 5493 women under 45 years of age sent to our cytological laboratory during 1988 . No anamnestic data concerning previous PAP smears of the women in both groups were available at the time of admission to the study.

To evaluate the incidence rate of cervical dysplasia, we one year later examined 722 of the prostitutes who had a negative PAP smear in 1988. As a 
control group we used PAP smears of 3162 women 11-13 months after negative cytology in 1988.

In 23 prostitutes and 42 non-prostitutes referred to our colposcopy clinic because of a PAP smear indicating mild to moderate dysplasia, colposcopically directed punch biopsies were sampled for histological diagnosis and HPV-DNA detection and typing.

PAP smear and colposcopy: Routine Papanicolaou smears were taken using a L-shaped spatula (SzalaySpatula, A. Graf, Steinach, Switzerland), reaching the endocervical and ectocervical region. Colposcopic examination was performed using a Zeiss (Germany) photocolposcope. When acetowhite regions were seen during the colposcopy a punch biopsy was performed. Biopsies were taken from the most suspect areas showing flat or everted condylomas, punctation or mosaic. Biopsy specimens then were fixed for at least 6 hours in buffered $(\mathrm{pH} 7 \cdot 0)$ formalin.

In situ hybridisation: $5 \mu \mathrm{m}$ thick sections of formalin fixed and paraffin embedded biopsies were mounted on amino-alcylisane pretreated slides, deparaffinised and then incubated for 10 minutes in $0.05 \%$ pronase in PBS at $37^{\circ} \mathrm{C}$. Then biotin-marked DNA probes for HPV 6/11, 16/18 and 31/33 were added (Enzo Biochem. Inc., New York, New York) and glass covered. Denaturation was performed in a microwave (HM 146, Elektra Bregenz, Schwaz, Austria) for 2 minutes at $240 \mathrm{~W}$ and 6 minutes at $120 \mathrm{~W}$ (microwave frequency: $2450 \mathrm{MHz}$ ). Hybridisation for 60 minutes at room temperature was followed by rinsing in PBS, addition of mouse-a-biotin (1:100 dilution in PBS) (Dakopatts, Carpintiera, USA) and incubation for 30 minutes at room temperature. Then the APAAP staining method was performed, using the bridge antibody rabbit-anti-mouse $I g, 1: 30$ dilution in PBS (Dakopatts, Carpintiera, USA), incubation time 30 minutes at room temperature. After rinsing in PBS the APAAP-complex (1:50 in PBS) (Dakopatts, Carpintiera, USA) was added and stored for 30 minutes at room temperature again. As a substrate we used AEC (2\% 3-Amino-9-ethylcarbazole and $3 \% \mathrm{H}_{2} \mathrm{O}_{2}$ in $0.05 \mathrm{M}$ Acetate Buffer), rinsed in aqua destillata and counterstained using hematoxylin for 30 seconds.

Statistical differences between the studied groups were calculated by chi square test. $P$ values $<0.05$ were regarded as statistically significant.

\section{Results}

Table 1 summarises the results of PAP smears of prostitutes and controls at the first examination. The frequency of positive cervical cytology in prostitutes $(6 \cdot 13 \%)$ was significantly higher than in the control
Table 1 PAP smear results at the first examination in prostitutes and controls

\begin{tabular}{lllll}
\hline & \multicolumn{2}{l}{$\begin{array}{l}\text { Prostitutes } \\
n=978\end{array}$} & \multicolumn{2}{c}{$\begin{array}{l}\text { Control group } \\
n=5493\end{array}$} \\
\cline { 2 - 5 } PAP smear result & $n$ & $\%$ & $n$ & $\%$ \\
\hline Mild to moderate dysplasia & 33 & 3.37 & 59 & 1.07 \\
Severe dysplasia & 23 & 2.35 & 20 & 0.36 \\
Invasive carcinoma & 4 & 0.41 & 0 & 0 \\
Total & 60 & 6.13 & 79 & 1.43 \\
\hline
\end{tabular}

group $(1.43 \%, p<0.0001)$. We evaluated $0.41 \%$ PAP smears indicating invasive carcinoma in prostitutes, whereas not a single one was found in the controls (table 1).

Of the prostitutes with a negative cytology 722 were re-examined one year later. Twenty-two (3.05\%) of them developed a PAP-smear indicating cervical dysplasia. In the control group $34(1.07 \%)$ of 3162 women showed a positive cervical cytology after the same observation period, demonstrating a significantly lower incidence of cervical dysplasia compared with the prostitute population $(\mathrm{p}<0.0001$ ) (table 2).

Histology of biopsy specimens of 23 prostitutes taken from suspect areas during colposcopy showed $18 \mathrm{CIN}$ I and $5 \mathrm{CIN}$ II. In 42 non prostitutes referred to our colposcopy clinic because of positive PAP smears histological findings revealed $30 \mathrm{CIN} \mathrm{I}$, $11 \mathrm{CIN}$ II and one CIN III.

Overall HPV detection rate in CIN lesions was equal in prostitutes $(47.8 \%)$ and controls $(50 \%)$. HPV types were equally distributed in the control group. In prostitutes we detected HPV 16/18 twice as often than HPV 6/11. In both groups HPV 31/33 infection showed a similar distribution. The difference in distribution of HPV types showed no statistical significance.

\section{Discussion}

Several authors described higher percentages of cervical dysplasia compared with a non-prostitute population. ${ }^{14} 15$ In agreement with these results, we found at the first examination a 4.3 times higher prevalence of cervical dysplasia compared with the

Table $2 P A P$ smear results in prostitutes and controls one year after negative cytology

\begin{tabular}{lcccc}
\hline & \multicolumn{2}{l}{$\begin{array}{l}\text { Prostitutes } \\
n=722\end{array}$} & \multicolumn{2}{l}{$\begin{array}{l}\text { Control group } \\
n=3162\end{array}$} \\
\cline { 2 - 5 } PAP smear result & $n$ & $\%$ & $n$ & $\%$ \\
\hline Mild to moderate dysplasia & 14 & 1.94 & 32 & 1.01 \\
Severe dysplasia & 8 & 1.11 & 2 & 0.06 \\
Invasive carcinoma & 0 & 0 & 0 & 0 \\
Total & 22 & 3.05 & 34 & 1.07 \\
\hline
\end{tabular}


control group. PAP smears indicating invasive carcinoma were found only in prostitutes. At the first routine cervical cytology all our patients had an unknown history of cervical dysplasia. Because of the lack of information in regard to the frequency of gynaecologic examinations, no conclusion could be drawn about the likelihood of the development of cervical dysplasia in both groups.

We therefore compared PAP smear results of prostitutes and the control group who had negative PAP smears at a previous examination 11 to 13 months before, to determine exactly the risk of picking up cervical dysplasia during one year. Compared with controls prostitutes showed a 2.8 fold higher incidence of PAP smears indicating cervical dysplasia.

Frequency of cervical dysplasia in prostitutes screened for the first time was 1.74 times higher than in prostitutes with a negative PAP smear one year before. In the control group frequency of detection of cervical dysplasia was 1.33 times higher at the first examination compared to PAP smear results one year after negative cytology. Thus, the difference in frequency of detection of cervical dysplasia between the first and second cytologic examination was markedly lower in the control group. These findings suggest that a higher percentage of prostitutes do not undergo regular cervical PAP smear by their own accord compared to controls.

The detection rate of HPV DNA in biopsy specimen of prostitutes and non-prostitutes was equal. Considering the fact that several authors make HPV infection responsible for the generation of CIN, the high incidence of cervical dysplasia in the prostitute population might suggest a more frequent HPV infection rate in this group. ${ }^{15-17}$ In our study, we did not find a higher HPV infection rate in prostitutes with cervical dysplasia. However, typing of HPV DNA revealed a more frequent infection rate with so called "high risk" HPV-types $16 / 18$ in prostitutes compared with the control group. The distribution of "low risk" HPV 6/11 and "high risk" $16 / 18$ and $31 / 33$ infection was approximately equal in the control group, whereas in prostitutes the infection rate with "low risk" HPV 6/11 was lower. These findings suggest that in addition to other well known risk factors like early first contact, a high number of changing partners and a high frequency of sexually transmitted diseases, HPV infection with predominantly high risk HPV-types might be an important factor in the frequent generation of cervical dysplasia in prostitutes. These multiple risk factors cause not only an increased frequency of PAP smears indicating cervical dysplasia or invasive carcinoma of the cervix at the first examination, but also a higher incidence of cervical dysplasia when regular cytologic examinations are performed.

Prostitutes seldom seem to undergo regular gynaecologic examinations and cervical PAP smears by their own request. Because of the significantly higher prevalence and pick-up rate of cervical dysplasia, in our opinion registered prostitutes, who are not examined by a private physician should undergo a cervical PAP smears twice a year in the public health service in addition to their regular screening for sexually transmitted diseases. ${ }^{18}$

The authors thank Mrs Andrea Steiner for excellent technical assistance. The study was supported by the "Medizinisch-wissenschaftlicher Fonds des Bürgermeisters der Bundeshauptstadt Wien" and Bender MedSystems, Austria.

Address for correspondence: G Gitsch, Second Department of Obstetrics and Gynecology, University of Vienna, Spitalgasse 23, A-1090, Vienna, Austria.

1 Sebastian JA. Cancer of the cervix - a sexually transmitted disease. Cytologic screening in a prostitute population. Am J Obstet Gynecol 1978;131:620-3.

2 Reid R, Stanhope CR, Herschman BR, Booth E, Phibbs GD, Smith JP. Genital warts and cervical cancer. I. Evidence of an association between subclinical papillomavirus infection and cervical malignancy. Cancer 1982;50:377-87.

3 Reid R, Greenberg, M, Jenson AB, et al. Sexually transmitted papillomaviral infections. I. The anatomic distribution and pathologic grade of neoplastic lesions associated with different viral types. Am J Obstet Gynecol 1987;156:212-22.

4 Reeves WC, Arosemena JR, Garcia M, et al. Genital human papillomavirus infection in Panama City prostitutes. J Infect Dis 1989;160:599-603.

5 Naguib SM, Lundin FE, Davis HJ. Relation of various epidemiologic factors to cervical cancer as determined by a screening program. Obstet Gynecol 1966;28:451-59.

6 Schuhmann RA, Hofmiller H, Mennicken C, Neumann G. Cytological smears in prostitutes. Geburtsh u Frauenheilk 1984;44:188-91.

7 Pereyra AJ. The relationship of sexual activity to cervical cancer. Obstet Gynecol 1961;17:154-9.

$8 \mathrm{Kopp} W$. The present examination schedule for prostitutes at the consulting office for venereal diseases, Public Health Service, Vienna. Off Gesundh-wes 1989;51:30-3.

9 Meanwell CA, Blackledge G, Gox MF, Maitland NJ. HPV 16 DNA in normal and malignant cervical epithelium: implications for the etiology and behaviour of cervical neoplasia. Lancet 1987;: : 703-7.

10 zur Hausen $\mathrm{H}$. Intracellular surveillance of persisting viral infections. Human genital cancer results from deficient cellular control of papillomavirus gene expression. Lancet 1986; ii:489-91.

11 Syrjänen K, de Villiers EM, Väyrynen M, et al. Cervical papillomavirus infection progressing to invasive cancer in less than three years. Lancet 1985;i:510-11.

12 Fuchs PG, Girardi, F, Pfister H. Human papillomavirus DNA in normal metaplastic, preneoplastic and neoplastic epithelia of the cervix uteri. Int $J$ Cancer 1988;41:41-5.

13 Moghissi KS, Mack HC, Porzak JP. Epidemiology of cervical cancer. Study of a prison population. Am J Obstet Gynecol 1968;100:607-14.

14 Keighley E. Carcinoma of the cervix among prostitutes in a women's prison. Br J Venereal Dis 1968;44:254-55.

15 Syrjänen $K$, Maentyjaervi $R$, Väyrynen $M$, et al. Human papillomavirus (HPV) infections involved in the neoplastic process of the uterine cervix as established by prospective follow-up of 513 women for two years. Eur J Gynaecol Oncol 1987;8:5-16.

16 Wendler $\mathrm{D}$. The frequency of human papillomavirus types 31 , 33 and 35 in cervical intraepithelial neoplasia. Geburtsh $u$ Frauenheilk 1990;50:110-12.

17 Göppinger A, Birmelin G, Hauser U, Ikenberg H. Clinical aspects of HPV 16/18 associated cervical lesions. Geburtsh u Frauenheilk 1990;50:106-9.

18 Abeyewickreme I. Cervical cytology screening in a sexually transmitted diseases clinic for the first time in Sri Lanka. Genitourin Med 1989;65:98-102.

Accepted for publication 2 September 1991 